\title{
Sleep in schizophrenia: time for closer attention ${ }^{\dagger}$
}

Sue Wilson and Spilios Argyropoulos

\section{Summary}

Recent sleep research has highlighted two specific anomalies in schizophrenia that have a proven impact on cognition. One is an abnormality of circadian rhythm, reported in this journal in two separate studies over the past year, and the other is the finding in electroencephalograms of reduced sleep spindles.

Declaration of interest

None.
Sue Wilson (pictured) is a senior research fellow in psychopharmacology in the Academic Unit of Psychiatry, University of Bristol and in the Neuropsychopharmacology Unit at Imperial College. She has expertise in the study of sleep in psychiatric disorders and the effects of psychotropic medication on sleep. Spilios Argyropoulos is a consultant psychiatrist at the South London and Maudsley NHS Foundation Trust and Honorary Senior Lecturer at the Institute of Psychiatry. His research has been in the psychopharmacology of antidepressants, including studies of sleep in depression and the effects of antidepressants.

Sleep problems in schizophrenia are all too familiar to mental health professionals and carers. Often sleep-inducing medication is prescribed or antipsychotics are chosen because of their enhanced sedative properties and their effects may persist into daytime. Sometimes sleep anomalies are seen as one of several problematic behaviours and patients may be castigated and lectured by clinicians and carers because of it. Publications in the past have highlighted sleep problems in schizophrenia, and more recently sleep disturbance has been identified as a contributing factor for the development of psychosis in young people at risk of schizophrenia. ${ }^{1}$ There has been a spate of research reports about sleep and schizophrenia in the past 2 or 3 years, both polysomnography and spectral analysis studies, that help to clarify the link between schizophrenia and sleep regulatory mechanisms, but of more direct interest to clinicians are some studies focusing on problems contributing to cognitive impairment, treatment success and engagement with psychological or behavioural therapies, and how these problems may be overcome. Two lines of recent research have highlighted some specific abnormalities in schizophrenia: (a) disturbance of circadian rhythm, and (b) changes in sleep architecture, especially sleep spindles.

\section{Circadian rhythm}

There are many clinical problems relating to sleep that present in individuals with schizophrenia but the most difficult to treat, from the point of view of psychiatrists, carers and project workers in mental health, is the tendency for these patients to sleep when the rest of the world is awake. In sleep medicine this is called circadian rhythm disorder, and occurs when the person's circadian sleep-wake drive from the suprachiasmatic nuclei of the hypothalamus is out of synchrony with the environment; the main influence on this innate circadian timing is light. Patients with psychosis may get very little daylight because some individuals tend to keep curtains closed during the day because of a perception of

†See pp. 308-316, this issue. threat from outside and others find the stimulation from general daytime hubbub distressing and may prefer to be awake in quieter periods. Many clinicians attribute the mis-timing either to sedative effects of medication or to negative symptoms of social withdrawal and apathy as part of the disease. However, there may also be an important link between this abnormality of sleep-wake rhythm in schizophrenia and other symptoms such as cognitive impairment, which could lead to targeted therapeutic interventions.

Two pivotal studies have been published in this journal recently that specifically describe sleeping and waking behaviour in schizophrenia. The most recent ${ }^{2}$ reports sleep-wake rhythms over 6 weeks using an objective measure, actigraphy, in community-dwelling people with schizophrenia. This involves the wearing of a simple wrist-worn device that measures movement continuously over days or weeks; only a little movement is registered during sleep and higher and variable movement during waking hours. They used a cohort of matched unemployed healthy volunteers as a control because they resembled the patients in the important factor of a lack of structure to their days. They found that there were indeed abnormalities in a substantial proportion of patients, with most showing longer sleep times than controls. The sleep phase in $50 \%$ of patients was out of synchrony with the environmental night-time, as was the rise and fall of melatonin (the biomarker for circadian rhythm). In general the patients had lower levels of daytime activity than the healthy unemployed group, and some also had an abnormally low amplitude of melatonin variation. The circadian misalignment was not related to clinical state or dose of antipsychotics, and it could not be explained simply by their everyday activity.

A similar finding of an abnormally low-amplitude restactivity cycle was found in an earlier paper in this journal ${ }^{3}$ in people with schizophrenia studied using actigraphy over 3 weeks, some of whom were in hospital or day care. In this study tasks of frontal lobe function were performed and those patients with a more normal rhythm performed better. Clinical status did not show any relation to cognitive performance, nor were measures of circadian rhythm related to the severity of either positive or negative symptoms. The authors conclude that a normal restactivity cycle with a high relative amplitude of day/night activity may be a prerequisite for adequate cognitive functioning in individuals with schizophrenia.

These two papers, with their convincing objective evidence of abnormal circadian rhythm, and the additional finding of cognitive function being lower in people with more abnormal rhythms, may be enough to justify the use of targeted systematic chronotherapeutic treatments (enhancing healthy sleep and daily activity, light therapy) to aid rehabilitation following an acute episode of the illness. 


\section{Sleep architecture: sleep spindles}

Sleep architecture, the structure of sleep and its electroencephalographic features, is less well researched in schizophrenia than in other psychiatric disorders such as depression. However, various non-specific abnormalities have been described, often in studies without adequate control groups, and more importantly in groups of patients taking various antipsychotic drugs. It is very difficult to undertake such studies in untreated patients without the confound of sleep disturbance due to distressing positive symptoms, and nearly all antipsychotic drugs affect a variety of brain neurotransmitters and in turn change sleep features. Funding for the study of sleep in schizophrenia is hard to come by, but research has been proceeding apace over the past 10 years, mostly in the USA with government funding.

Sleep spindles, short waxing and waning runs of oscillations at about 12-14 per second, were one of the earliest patterns to be identified in human sleep and can be recorded easily from the scalp over the whole night in light sleep, and they diminish in deep sleep and in rapid-eye movement sleep (Fig. 1). Their occurrence is approximately inversely related to the large slow waves that occur during deep sleep, and both are generated by thalamocortical oscillations. Although the electrophysiology of these spindles has been well-defined for many years, their functional relationship to learning and memory has only come to light over the past 10 years, with much of this basic sleep research coming from Germany. Now we know that the number and type of sleep spindles is related to learning ability, that they increase when learning has taken place during the preceding day, that this increase is related to sleep-dependent improvement in the learning task and that they may reflect efficient thalamocortical communication. ${ }^{4}$

Three important studies of sleep spindles and their relationship to cognition in schizophrenia have been published in the past 3 years, from cohorts in Madison, ${ }^{5}$ Pittsburgh $^{6}$ and Boston. ${ }^{7}$ Ferrarelli et al showed that sleep spindles were reduced in amplitude and duration in 49 participants with schizophrenia who were taking medication in comparison with 44 matched controls, and also in comparison with a group of 20 nonschizophrenia patients receiving antipsychotic medication. ${ }^{5}$ This is interesting because some antipsychotics change sleep parameters like slow waves and sleep spindles, even in 'normal' controls. This study shows that there is a reduction in spindles in only the participants with schizophrenia and not the other participants on antipsychotics, thus the reduction is not explained by the effect of antipsychotic drugs. Keshavan et als study from Pittsburgh ${ }^{6}$ showed that a group of 27 newly diagnosed untreated patients had reductions in spindles that correlated with lower performance on frontal cognitive tasks. The Boston group ${ }^{7}$ had previously reported that patients with schizophrenia, when compared with controls, did not show the normal improvements in a motor task (a finger-tapping sequence) after a night's sleep. Their subsequent study evaluated sleep spindles overnight, after learning of this task in 27 participants with schizophrenia who were taking medication and again looked at morning improvement. The lower the spindle number and density the smaller were the improvements in the task. These three studies, using a relatively easy and inexpensive (compared with neuroimaging) method, demonstrate a reliable neurobiological marker in schizophrenia that indicates disrupted thalamocortical activity, and which suggests a new treatment target.

\section{Implications}

The reports from both of the two areas of sleep research above, circadian rhythm disturbance and sleep electroencephalograms,

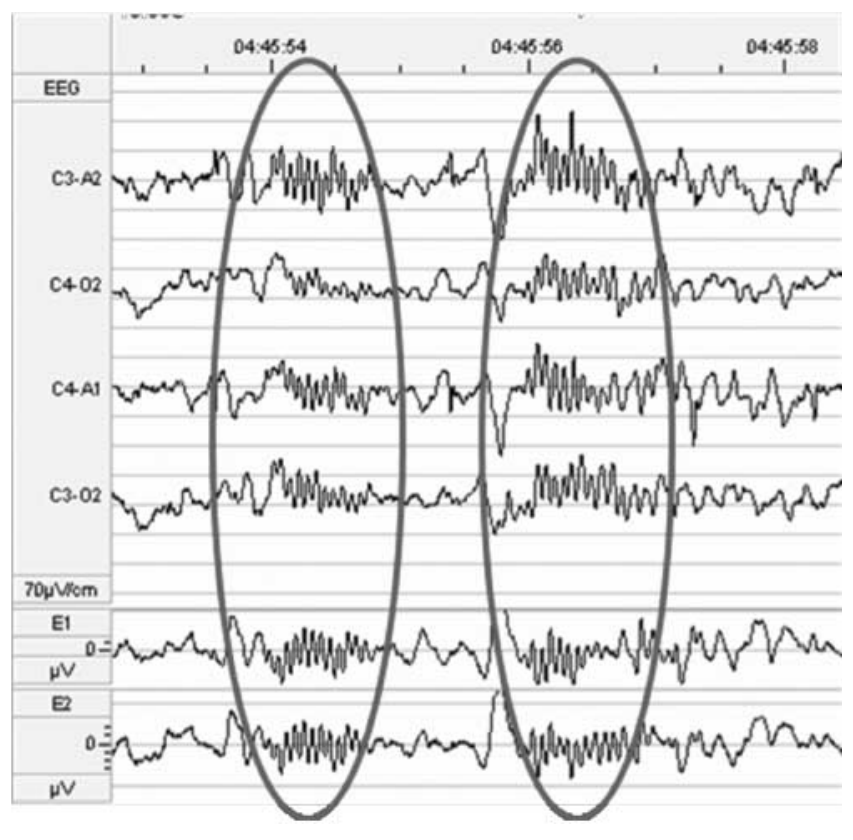

Fig. 1 Electroencephalogram (EEG) showing sleep spindles in stage two of sleep

and their links with cognition, are welcome strides forward in our understanding of the neurobiological processes underlying schizophrenia. It may be that cognitive improvement in people with schizophrenia depends on improving sleep parameters. We await with interest the development of these promising lines of research.

Sue Wilson, PhD, Academic Unit of Psychiatry, University of Bristol, and Neuropsychopharmacology Unit, Imperial College, London; Spilios Argyropoulos, MSc, MRCPsych, PhD, South London and Maudsley NHS Foundation Trust, and Section of Neurobiology of Psychosis, Institute of Psychiatry, London, UK

Correspondence: Sue Wilson, Academic Unit of Psychiatry, School of Social and Community Medicine, University of Bristol, Room BF2, Oakfield House, Bristol BS8 2BN, UK. Email: sue.wilson@bristol.ac.uk

First received 2 Dec 2011, final revision 2 Dec 2011, accepted 13 Feb 2012

\section{References}

1 Ruhrmann S, Schultze-Lutter F, Salokangas RKR, Heinimaa M, Linszen D, Dingemans $P$, et al. Prediction of psychosis in adolescents and young adults at high risk: results from the prospective European prediction of psychosis study. Arch Gen Psychiatry 2010; 67: 241-51.

2 Wulff K, Dijk D-J, Middleton B, Foster RG, Joyce EM. Sleep and circadian rhythm disruption in schizophrenia. Br J Psychiatry 2012; 200: 308-16.

3 Bromundt V, Köster M, Georgiev-Kill A, Opwis K, Wirz-Justice A, Stoppe G, et al. Sleep-wake cycles and cognitive functioning in schizophrenia. Br J Psychiatry 2011; 198: 269-76.

4 Fogel SM, Smith CT. The function of the sleep spindle: a physiological index of intelligence and a mechanism for sleep-dependent memory consolidation. Neurosci Biobehav Rev 2011; 35: 1154-65.

5 Ferrarelli F, Peterson MJ, Sarasso S, Riedner BA, Murphy MJ, Benca RM, et al. Thalamic dysfunction in schizophrenia suggested by whole-night deficits in slow and fast spindles. Am J Psychiatry 2010; 167: 1339-48.

6 Keshavan MS, Montrose DM, Miewald JM, Jindal RD. Sleep correlates of cognition in early course psychotic disorders. Schizophr Res 2011; 131: 231-4.

7 Wamsley EJ, Tucker MA, Shinn AK, Ono KE, McKinley SK, Ely AV, et al. Reduced sleep spindles and spindle coherence in schizophrenia: mechanisms of impaired memory consolidation? Biol Psychiatry 2012; 71: 154-61. 